\title{
Alta prevalencia de disfunción tiroidea en pacientes psiquiátricos hospitalizados
}

\author{
Sergio Valdivieso $\mathrm{F}^{\mathbf{1}}$, Cristóbal Krippera, José Antonio Ivelic ${ }^{1 \mathrm{~b}}$, \\ Carlos Fardella², Sergio Gloger ${ }^{3}$, Danilo Quiroz ${ }^{3}$. \\ High prevalence \\ of thyroid dysfunction \\ among psychiatric inpatients
}

Background: Thyroid dysfunction is frecuent in psychiatric outpatients and in the general Chilean population but there is no information about the prevalence of thyroid diseases in Chilean psychiatric inpatients. Aim: To retrospectively assess the frequency of thyroidal diseases in psychiatric inpatients. Material and Methods: Clinical charts and thyroid assessment of 241 psychiatric inpatients (147 women, mean age $33 \pm 16$ years) attended in a University Psychiatric Clinic, were reviewed. Psychiatric diagnosis at discharge was made according to DSM IV criteria and endocrine diagnosis was made based on international criteria. Results: Forty nine patients (20.7\%) had thyroid abnormalities. Forty four patients had hypothyroidism (18.3\%) and five had hyperthyroidism (2.35\%). No specific associations were found between gender or psychiatric diagnosis and endocrine abnormalities. Conclusions: In this sample, the prevalence of thyroid abnormalities was similar to other reports in psychiatric inpatiens and higher than in the general population in Chile (Rev Méd Chile 2006; 134: 623-8).

(Key w ords: Hospitals, Psychiatric; Mental disorders; Thyroid diseases)

Recibido el 2 de agosto, 2005. Aceptado el 17 de noviembre, 2005.

${ }^{1}$ Departamento de Psiquiatría, Facultad de Medicina, Pontificia Universidad Católica de Chile. ${ }^{2}$ Departamento de Endocrinología, Facultad de Medicina, Pontificia Universidad Católica de Chile. ${ }^{3}$ PSICOMEDICA.

anterno, Departamento de Psiquiatría, Facultad de Medicina, Pontificia Universidad Católica de Chile.

bMédico residente

T a adecuada función del eje hipotálamo-hipófisis-tiroides se traduce en la producción de hormona tiroidea necesaria para mantener el funcionamiento normal de variados órganos y sistemas, entre ellos el sistema nervioso central (SNC). A

Correspondencia a: Sergio Valdivieso F. Clínica UC San Carlos. Camino El Alba 12351, Las Condes, Santiago, Chile. Fax: 7548878. E mail: svaldivi@med.puc.cl este nivel, el déficit de hormona tiroidea produce síntomas como astenia psicomotora, somnolencia, pérdida de memoria y dificultad de concentración, síntomas que también se presentan en el curso de determinados trastornos psiquiátricos. De allí la importancia de una evaluación tiroidea sistemática en todo paciente que presente síntomas anímicos 0 ansiosos.

Los trastornos psiquiátricos son actualmente una de las patologías de mayor prevalencia en el 
mundo ${ }^{1}$. En nuestro país se estima que estas enfermedades pueden afectar hasta a $36 \%$ de la población total ${ }^{2,3}$. Por su parte, la prevalencia de enfermedades tiroideas también es alta, afectando de 5 a $12 \%$ de la población general ${ }^{4}$. En Chile se ha descrito una prevalencia de 7,5\% de trastornos tiroideos en pacientes que concurrían a un control sano de salud ${ }^{5}$.

Existen numerosos reportes que asocian patologías tiroideas con enfermedades neuropsiquiátricas ${ }^{6-9}$. La principal asociación que se ha encontrado es entre el hipotiroidismo y depresión 7,9 , describiéndose en Chile una prevalencia de hipotimoidismo de $12 \%$ en pacientes consultantes en un centro de psiquiatría ambulatoria y de $23 \%$ en pacientes con depresión refractaria a tratamiento ${ }^{10,11}$. Estudios en el extranjero indican que la prevalencia de trastomos timideos en pacientes psiquiátricos hospitalizados va desde $6 \%$ a 49\%12-19. Actualmente en Chile no existen estudios que describan esta asociación. El objetivo de este estudio es describir la frecuencia y tipo de disfunción trmidea en pacientes psiquiátricos hospitalizados.

\section{PaCIENTES y MÉTOdos}

Pacientes. El estudio fue realizado en base a las fichas clínicas de pacientes hospitalizados en el Servicio de Psiquiatría de la Clínica San Carlos de la Pontificia Universidad Católica de Chile. Se trata de un servicio universitario de psiquiatría, que cuenta con 20 camas para pacientes con patología psiquiátrica aguda, habiéndose hospitalizado en sus tres años de funcionamiento (peńodo 2001-2004) un total aproximado de 800 pacientes. Se realizó un muestreo aleatorio simple mediante el cual se seleccionaron un total de 312 fichas. El cálculo muestral fue obtenido a través del programa epi info 6.0.

Al momento del ingreso al hospital, 25,7\% de los pacientes se encontraban casados, $53,5 \%$ solteros, $8,7 \%$ separados, $3,3 \%$ era viudo o convivía y el $8,7 \%$ restante no presentó datos sobre su estado civil. El 19\% de la muestra tenía estudios escolares en curso o incompletos, $15,3 \%$ estudios escolares completos, $24,8 \%$ presentaba estudios superiores en curso o incompletos y 34,4\% presentaba estudios superiores completos. El 6,2\% de la muestra no aportaba datos sobre nivel educacional.

La extracción de datos fue realizada por medio de una encuesta en la cual se consideraron los diagnósticos psiquiátricos consignados en el informe de egreso de la hospitalización (epicrisis). En los casos en los que no se consignaba el diagnóstico al alta, se incluyeron los diagnósticos de ingreso. Para el estudio de la prevalencia de disfunción tiroidea según diagnóstico psiquiátrico, se consideró el diagnóstico principal consignado al egreso. Cuando existían dudas, se consideró el diagnóstico que había motivado el ingreso. En los pacientes que fueron hospitalizados más de una vez, se consideraron los datos presentes en la última hospitalización que contuviera mediciones tiroideas. Los medicamentos considerados en el estudio son aquellos consignados como tratamiento previo al momento del ingreso. Sólo se consideraron aquellos pacientes que presenten mediciones tiroideas realizadas dentro de las primeras $48 \mathrm{~h}$ tras registrado el ingreso del paciente. En todos los casos se consignaron los niveles séricos de triyodotironina (T3), tiroxina (T4), tiroxina libre (T4L), tirotropina (TSH), así como la presencia de anticuerpos antiperoxidasa (A-TPO) y antitiroglobulina (A-Tg). La presencia de bocio no se consignó en este estudio.

Diagnóstico de hipotiroidismo. Se realizó mediante el antecedente de diagnóstico de hipotiroidismo 0 la presencia al ingreso de TSH $>10 \mathrm{uUI} / \mathrm{ml}$. Hipotiroidismo subclínico se entiende presente en aquellos pacientes con valores de TSH entre 4,2 y $10 \mathrm{uUI} / \mathrm{ml}$ con hormonas periféricas normales.

Diagnóstico de tirotoxicosis. Se realizó ante la presencia de valores de niveles plasmáticos de T3 $>180 \mathrm{ng} / \mathrm{dl}$ (normalidad 80-180 ng/dl) o T4L >1,8 $\mathrm{ng} / \mathrm{dl}$ (normalidad: 0,9-1,8 ng/dl) en presencia de TSH $<0,4 \mathrm{uUI} / \mathrm{ml}$ (normalidad 0,4-4,0). Dado que no se realizó captación de I-131, no es posible determinar si los pacientes eran realmente hipertiroideos, por lo que decidimos catalogarlos como cursando un cuadro de tirotoxicosis.

Método. La determinación de los niveles de hormonas tiroideas, así como de los diferentes anticuerpos antitimoideos, se realizó en el Servicio de Laboratorios Clínicos de la Universidad Católica de Chile. Las hormonas TSH, T3, T4, y T4L fueron medidas en el equipo automático ACS-180 (Chiron Diagnostics) por inmunoensayos quimioluminiscentes, bajo control de calidad interno y externo del Colegio de Patólogos Americano (CAP). La determinación de 
TSH fue realizada por un ensayo inmunométrico (ICMA) de tercera generación, con una sensibilidad funcional de alrededor de $0,01 \mathrm{uUI} / \mathrm{ml}$ y un coeficiente de variación (CV) interensayo de 4,5\% para un nivel de 2,6 uUI/ml. Las concentraciones de T3, T4 y T4L fueron medidas por inmunoensayo competitivos, con CV interensayo de 6,0, 5,2, y 8,2\% para concentraciones de T3, T4 y T4L de $141 \mathrm{ng} / \mathrm{dl}, 7,3$ $\mathrm{ug} / \mathrm{dl}, 1,15 \mathrm{ng} / \mathrm{dl}$, respectivamente. Los anticuerpos antimicrosomales fueron determinados por hemaglutinación (Murex Diagnostic Limited, Inglaterra). Los niveles de A-Tg y A-TPO fueron determinados por inmunoensayos quimioluminescentes en un equipo automático (Immulite, DPC).

\section{Resultados}

De las 312 fichas clínicas analizadas, 71 fueron descartadas por presentar datos incompletos (68 sin mediciones de TSH y 3 por ausencia de diagnóstico psiquiátrico, de éstas, 4 (5,1\%) presentaron antecedente de hipotiroidismo). De un total de 241 sujetos, 94 eran hombres (39\%) y 147 mujeres (61\%), con edades entre 9 y 90 años y un promedio de 33,48 $\pm 16,22$ años.

Se diagnosticó un hipotiroidismo en 44/241 pacientes (18,3\%). En 28 casos existía el antecedente de hipotiroidismo $(11,61 \%)$ y se encontraban en terapia de sustitución con hormona tiroidea. De ellos, al ingreso 11 se encontraban eutiroideos, 6 persistían con hipotiroidismo subclínico y 4 presentaban valores de TSH bajo 0,4 uUI/ml. Este grupo fue apartado para el análisis de función timoidea de ingreso. En 16 casos (6,6\%) se diagnosticó hipotiroidismo de novo, de los cuales 14 correspondían a hipotiroidismo subclínico y 2 eran pacientes portadores de un hipotiroidismo sintomático.

Se diagnosticó un hipertiroidismo en 5/241 casos (2,35\%). La Tabla 1 describe la presencia de disfunción tiroidea calculada por sexo, la cual no resultó ser significativamente diferente. Tampoco se demostró una mayor presencia de alteraciones tiroideas en una patología psiquiátrica determinada, tal distribución se presenta en la Tabla 2 . Respecto de los anticuerpos antitimideos, éstos se

Tabla 1. D iffunción tiroidea según sexo en pacientes psiquiátricos hospitalizados ${ }^{1}$

\begin{tabular}{|lrrrrrr|}
\hline Disfunción tiroidea & Hombres & $\mathrm{n}=94$ & Mujeres & $\mathrm{n}=147$ & \multicolumn{2}{c|}{ Total $\mathrm{n}=241$} \\
\hline Hipotiroidismo & 16 & $(17 \%)$ & 28 & $(19 \%)$ & $44 \quad(18,2 \%)$ \\
Hipertiroidismo & 0 & & 5 & $(3,4 \%)$ & 5 & $(2 \%)$ \\
Bocio eutiroideo & 0 & 1 & $(0,6 \%)$ & 1 & $(0,4 \%)$ \\
Total & 16 & $(17 \%)$ & 34 & $(23,1 \%)$ & $50 \quad(20,7 \%)$ \\
\hline
\end{tabular}

${ }^{1}$ No hubo diferencias estadísticamente significativas entre hombres y mujeres.

Tabla 2. Prevalencia de disfunción tiroidea según diagnóstico en pacientes psiquiátricos hospitalizados ${ }^{1}$

\begin{tabular}{|c|c|c|c|c|c|}
\hline \multirow[t]{2}{*}{ Diagnóstico } & \multirow[t]{2}{*}{$\mathrm{n}$} & \multicolumn{2}{|c|}{ Hipotiroidismo } & \multirow{2}{*}{$\begin{array}{c}\text { Hipertiroidismo } \\
\mathrm{n} \quad(\%)\end{array}$} & Total con disfunción tiroidea \\
\hline & & $\mathrm{n}$ & $(\%)$ & & $\mathrm{n} \quad(\%)$ \\
\hline Psicosis & 15 & 1 & $(6,6 \%)$ & 0 & $1 \quad(6,6 \%)$ \\
\hline T. A. Unipolar & 87 & 14 & $(16 \%)$ & $2(2,2 \%)$ & $16(18,3 \%)$ \\
\hline T. A. Bipolar & 95 & 23 & $(24,4 \%)$ & $2(2,1 \%)$ & $25 \quad(26,3 \%)$ \\
\hline T. Ansiedad & 1 & 0 & & 0 & 0 \\
\hline T. Orgánico & 6 & 1 & $(16,6 \%)$ & 0 & $1 \quad(16,6 \%)$ \\
\hline T. Alimentario & 4 & 0 & & 0 & 0 \\
\hline T. Personalidad & 15 & 4 & $(26,6 \%)$ & 0 & $4 \quad(26,6 \%)$ \\
\hline Adicciones & 18 & 1 & $(5,5 \%)$ & $1 \quad(5,5 \%)$ & $(11 \%)$ \\
\hline Total & 241 & 44 & $(18,3 \%)$ & $5(2,35 \%)$ & $49(20,33 \%)$ \\
\hline
\end{tabular}

${ }^{1}$ No hubo diferencias estadísticamente significativas en la prevalencia de patología tiroidea según diagnóstico. 
solicitaron sólo en 19 pacientes, razón por la cual no fueron analizados.

\section{DisCUSIÓN}

En este estudio retrospectivo se encontró una alta frecuencia de alteraciones tiroideas en pacientes hospitalizados por un trastorno psiquiátrico. Según nuestro conocimiento, éste sería el primer estudio en Chile que estudia la presencia de patología tiroidea en este tipo de población. La prevalencia de alteraciones tiroideas en pacientes psiquiátricos hospitalizados varía entre $6 \%$ y $49 \%{ }^{12-19}$. Lo más característico es el síndrome de enfermedad eutiroidea (euthyroid sick syndrome), en el cual hay una hipertiroxinemia que habitualmente se normaliza al disminuir los síntomas ${ }^{20}$.

La mayor parte de las alteraciones encontradas en este estudio corresponden a hipotiroidismo (18,3\%), con una proporción de 9/1 respecto del hipertiroidismo. En la literatura se señala que entre $1 \%$ y $4 \%$ de los pacientes con trastornos afectivos presentan hipotiroidismo clínico y entre $4 \%$ y $40 \%$, hipotiroidismo subclínico ${ }^{20}$. En la muestra de este estudio $80 \%$ de los pacientes tuvieron el diagnóstico de trastorno afectivo unipolar y bipolar, lo cual podría explicar estos hallazgos. La presencia de alteraciones sutiles de la función tiroidea tiene significación clínica, ya que se asocia a mayor incidencia de depresión y trastorno de pánico, disfunción cognitiva y menor respuesta a tratamiento antidepresivo. Se ha encontrado que la frecuencia de depresión mayor a lo largo de la vida es significativamente más alta en aquellos pacientes con hipotiroidismo subclínico que en aquellos en los que no existe disfunción tiroidea ${ }^{21,22}$. En el caso del trastorno bipolar, en el último tiempo se ha descrito que uno de los factores que predisponen a ser ciclador rápido (cuatro o más episodios afectivos al año) es la comorbilidad con hipotiroidismo, con cifras que llegan a 92\% de hipotiroidismo en cicladores rápidos versus $32 \%$ en cicladores más lentos ${ }^{23}$. Este hecho ha llevado a algunos investigadores a la utilización de T4 en pacientes bipolares refractarios al tratamiento habitual con buenos resultados. En dos estudios se utilizaron dosis suprafisiológicas de T4 con efectos colaterales mínimos 24,25 . La experiencia clínica de los autores coincide con la impresión de que existiría un subgrupo de pacientes con trastornos afectivos graves que podrían beneficiarse de dosis suprafisiológicas de hormonas tiroideas, efecto cuyo mecanismo aún no es claro.

Como era esperable, el grupo de pacientes hospitalizados corresponden al espectro grave de la patología psiquiátrica, con comorbilidad frecuente, por lo que habitualmente requieren para su adecuado tratamiento del uso de varios psicofármacos. En esta muestra 43\% estaban utilizando antidepresivos, 43\% ansiolíticos, 22\% estabilizadores del ánimo, 23\% antipsicóticos y $4 \%$ hipnóticos y psicoestimulantes. Más de $90 \%$ de los pacientes tenía dos o más fármacos. Existen evidencias del efecto del litio a distintos niveles, entre ellos, la inhibición de la conversión de T4 a T3, la captación de yodo, la producción y secreción de hormona tiroidea ${ }^{26,27}$. Especialmente en mujeres pueden elevarse los anticuerpos antitimideos y desarrollarse un hipotimidismo hasta en $30 \%$ de los casos. La carbamazepina produce una disminución de hormonas tiroideas y una menor respuesta de TSH a TRH ${ }^{28-31}$. Con respecto a los antidepresivos, se ha reportado aumento de TSH y disminución de T3 y T4 con maprotilina, fluvoxamina y un aumento del clearence de T4 con sertralina ${ }^{32-35}$.

Otra patología importante a considerar es el abuso de alcohol. Veinticinco por ciento de los pacientes de este estudio tenían abuso y dependencia de alcohol. Se ha descrito que los pacientes alcohólicos con daño hepático tienen bajos niveles de T3 (probablemente por una baja conversión de T4 a T3) y aumento de TSH basal ${ }^{36}$. Por otra parte, es frecuente una baja respuesta de TSH a la estimulación con TRH en pacientes sin daño hepático crónico, sugiriéndose que podría ser un marcador de rasgo, ya que es una alteración que persiste durante la abstinencia ${ }^{37}$.

Al comparar las perturbaciones tiroideas de esta población con el estudio realizado en Chile por Fardella y cols ${ }^{10}$ en pacientes psiquiátricos ambulatorios, se observó una menor frecuencia de hipertiroidismo en los pacientes hospitalizados, lo cual se explica por el hecho de que la muestra ambulatoria estuvo constituida en $40 \%$ por pacientes con trastomo de pánico, quienes a su vez concentraron la mayor frecuencia de hipertiroidismo. Existen algunos estudios en la literatura que confirman la asociación entre trastorno de pánico, bocio e hipertiroidismo ${ }^{38,39}$. En la actualidad, el tratamiento del trastorno de pánico es 
ambulatorio y la indicación de hospitalización es excepcional, lo que explica que no se consigne como diagnóstico principal. En relación al hipotiroidismo, la prevalencia en pacientes psiquiátricos ambulatorios también fue mayor a la encontrada en pacientes psiquiátricos hospitalizados.

No se encontró una diferencia significativa de la frecuencia de alteraciones tiroideas entre los distintos diagnósticos, aun cuando no es posible sacar conclusiones definitivas, ya que como se mencionó, la muestra estuvo constituida mayoritariamente por trastornos afectivos. Llama la atención una alta frecuencia de disfunción tiroidea en pacientes con trastorno de la personalidad, lo que no ha sido descrito en la literatura. Lo anterior podría asociarse a una alta comorbilidad de estos trastornos con depresión o a la utilización frecuente en estos casos de estabilizadores del ánimo.

El presente estudio tiene algunas limitaciones. Por tratarse de una revisión retrospectiva de fichas clínicas, los datos de los pacientes estaban incomple-

\section{REFERENCIAS}

1. Kessier RC, McGonagie KA, Zhao S, Neison CB, Hughes M, EshizMAN S ET AL. Lifetime and 12-month prevalence of DSM $\mathrm{m}-\mathrm{R}$ psychiatric disorders in the United States: results from the National Comorbidity Study. Arch Gen Psychiatry 1994; 345: 8-19.

2. Vicente B, Rioseco P, Vielma M, Uribe M, Boggiano G, Torres S. Prevalencia de vida de algunos trastornos psiquiátricos en la provincia de Concepción. Rev Psiquiatría 1992; IX: 1050-60.

3. Araya R, Rojas G, Fritsch R, Acuña J, Lewis G. Common mental disorders in Santiago, Chile: Prevalence and socio-demographic correlates. $\mathrm{Br}$ J Psychiatry 2001; 178: 228-33.

4. Vanderpump MPJ, Tunbridge WMG, Frech JM, Appieton D, Bates D, Ciark F et al. The incidente of thymoid disorders in the community: a twenty years followup of the Whickham Survey. Clin Endocrinol 1995; 43: 55-68.

5. Fardella C, Poggi H, Gloger S, Rojas A, Velásquez C, Barroilet S Et al. Alta prevalencia de enfermedad tiroidea en sujetos aparentemente sanos que concurren a control de salud. Rev Méd Chile 2001; 129: 155-60.

6. Quiroz D, Gloger S, Valdivieso S, Ivelic J, Fardelia C. Trastornos del ánimo, psicofármacos y tiroides. Rev Méd Chile 2004; 132: 1413-24. tos en un porcentaje importante de los sujetos. Pese a lo anterior, el tamaño de la muestra continuó siendo representativo. Por otra parte, es una muestra con alta comorbilidad y múltiples psicofármacos, por lo que pueden coexistir varios factores que influyen en la disfunción timidea, lo que impide saber con más precisión la relación entre ésta y el tipo de patología psiquiátrica. Se debe tener precaución con la generalización de estos resultados, ya que este grupo de pacientes es particularmente grave y de un nivel socioeconómico medio-alto.

En resumen, este estudio demuestra una alta frecuencia de disfunción tiroidea en pacientes psiquiátricos hospitalizados, siendo el hipotiroidismo subclínico la alteración más frecuente. Considerando que los pacientes psiquiátricos graves tienen numerosos factores que pueden potencialmente perturbar la función tiroidea a distintos niveles, es recomendable realizar de rutina un estudio de su nivel hormonal, lo que puede ayudar a evitar complicaciones de la patología psiquiátrica.

7. Prange AJ, Haggerty JJ, Browne JL, Rice JD. Marginal hypothyroidism in mental illness: Preliminary assesments of prevalence and significance. En: Bunney WE Jr, Hippius H, Laakmann G, Schmauss M, ed. Neuropsychopharmacology Berlin: Springer-Verlag, 1990; 352-61.

8. Tappy L, Randin JP, Schwed P, Wertheimer J, LemarCHAND-BERAUD T. Prevalence of thyroid disorders in psychogeriatric inpatients. A possible relationship of hypothyroidism with neurotic depression but not with dementia. J Am Geriatr Soc 1987; 35: 526-31.

9. Jofre RT, LevitT AJ. Major depression and subclinical (grade 2) hypothyroidism. Psychoneuroendocrinology 1992; 17: 215-21.

10. Fardelia C, Gloger S, Figueroa R, Santis R, Gajardo C, Salgado C ET al. High prevalence of thyroid abnormalities in a Chilean psychiatric population. J Endocrinol Invest 2000; 23: 102-6.

11. Howland RH. Thyroid dysfunction in refractory depresión: implications for patho-physiology and treatment. J Clin Psychiatry 1993; 54: 47-54.

12. Cohen KL, Swigar ME. Thyroid function screening in psychiatric patients. JAMA 1979; 242: 254-7.

13. WeINBERG AD, Katzell TD. Thyroid and adrenal function among psychiatric patients. Lancet 1977; 1: 1104-5.

14. Spratt DI, Pont A, Milier MB, McDougall IR, Bayer MF, McLaughuin WT. Hyperthyroxinemia in pa- 
tients with acute psychiatric disorders. Am J Med 1982; 73: 41-8.

15. MorLey JE, SHAFER RB. Thyroid function screening in new psychiatric admissions. Arch Intern Med 1982; 142: 591-3.

16. Roca RP, Biackman MR, Ackerley MB, Harman SM, GREGERMAN RI. Thyroid hormone elevations during acute psychiatric illness: relationship to severity and distinction from hyperthyroidism. Endocr Res 1990; 16: 415-47.

17. Chopra IJ, Solomon DH, Huang TS. Serum thymotropin in hospitalized psychiatric patients: evidence for hyperthyrotropinemia as measured by an ultrasensitive thyrotropin assay. Metabolism 1990; 39: 538-43.

18. Levy RP, Jensen JB, Laus VG, Agle DP, Engel IM. Serum thyroid hormone abnormalities in psychiatric disease. Metabolism 1981; 30: 1060-4.

19. McLarty DG, Ratclffe WA, RatclfFe JG, Shimmins JG, GOLDBERG A. A study of thyroid function in psychiatric in-patients. Br J Psychiatry 1978; 133: 211-8.

20. LARSEN PR, IngBar SH. The thyroid gland. E: Wilson JD and Foster DW, eds. Textbook of Endocrinology. Philadelphia, PA, WB Saunders. 1992: 357-487.

21. O'Connor D, Gwirtman H, Loosen P. Thymid Function in Psychiatric Disorders. En: O.M. Wolkowitz and A.J. Rothschild, eds. Psychoneuroendocrinology: the scientific basis of clinical practice. American Psychiatric Press, Inc. 2003: 361-418.

22. Haggerty JJ Jr, Stern RA, Mason GA, Beckwith J, Morey CE, Pranje AJ Jr. Subclinical hypothyroidism: a modifiable risk factor for depression? Am J Psychiatry 1993; 150: 508-10.

23. Cowdry RW, WeHR TA, Zis AP, Goodwin FK. Thyroid abnormalities associated with rapid-cycling bipolar illness. Arch Gen Psychiatry 1983; 40: 414-20.

24. Bauer MS, Whybrow PC. Rapid cycling bipolar affective disorder II: treatment of refractory rapid cycling with high-dose levothyroxine: a preliminary study. Arch Gen Psychiatry 1990; 47: 435-40.

25. Baumgartner A, Bauer M, Hellweg R. Treatment of intractable non-rapid cycling bipolar affective disorder with high-dose thyroxine: an open clinical trial. Neuropsychopharmachology 1994a; 10: 183-9.

26. Burrow GN, Burke WR, HimmelHoch JM, SPencer RP, HERSHMAN JM. Effect of lithium on thyroid function. J Clin Endocrinol Metab 1971; 32: 647-52.

27. Carlson HE, Temple R, Robbins J. Effect of lithium on thyroxine disappearence in man. J Clin Endocrinol Metab 1973; 36: 1249-54.
28. Roy-Byrne PP, Joffe RT, Uhde TW, Post RM. Carbamazepine and thyroid function in affectively ill patients. Clinical and theoretical implications. Arch Gen Psychiatry 1984; 41: 1150-3.

29. Herman R, Obarzanek E, Mikalauskas KM, Post RM, JiMERSON DC. The effects of carbamazepine on resting metabolic rate and thyroid function in depressed patients. Biol Psychiatry 1991; 29: 779-88.

30. JofFe RT, Gol PW, UhDe TW, Post RM. The effects of carbamazepine on the thyrotropin response to thyrotropin-releasing hormone. Psychiatry Res 1984; 12: 161-6.

31. ERICSSON UB, BJerRe I, Forsgren M. Thyroglobulin and thyroid hormones in patients on long-term treatment with phenytoin, carbamazepine, and valproic acid. Epilepsia 1985; 26: 594-6.

32. Hoeflich G, Kasper S, Danos P, Schmidt R. Thyroid hormones, body temperature, and antidepressant therapy. Biol Psychiatry 1992; 31: 859-62.

33. Brady KT, LydiaRd RB, KelnNer CH, JofFe R, Laird LK, Morton WA ET AL. A comparison of the effects of imipramine and fluvoxamine on the thyroid axis. Biol Psychiatry 1994; 36: 778-9.

34. Baumgartner A, Dubey ko M, Campos-Barros A, Eravci M, MeINHOLD H. Subchronic administration of fluoxetine to rats affects triiodothyronine production and deiodination in regions of the cortex and in the limbic forebrain. Brain Res 1994; 635: 68-74.

35. McCowen KC, Garber JR, Spark R. Elevated serum thyrotropin in thyroxine-treated patients with hypothyroidism give sertraline (letter). $\mathrm{N}$ Engl J Med 1997; 337: 1010-11.

36. HepNER GW, Chopra U. Serum thyroid hormone levels in patients with liver disease. Arch Intern Med 1979; 139: 1117-20.

37. Muelier N, Hoene M, Kiein HE, Nieberie G, KapfhamMER HP, MAY F ET AL. Endocrinological studies in alcoholics during withdrawal and after abstinence. Psychoneuroendocrinology 1989; 14: 113-23.

38. Chiovato L, Marino M, Perugi G, Fiore E, Montaneш L, LAPI P. Chronic recurrent stress due to panic disorder does not precipitate Graves' disease. J Endocrinol Invest 1998; 21: 758-64.

39. Lesser IM, Rubin RT, Lydiard RB, Swinson R, PecKNoid J. Past and current thyroid function in subjects with panic disorder. J Clin Psychiatry 1987; 48: 473-6.

Agradecimientos

Luis Villarroel. Estadístico Depto. Salud Pública, Facultad de Medicina PUC. 